\title{
LOS PARES EXTERNOS INTERNACIONALES EVALUADORES DE LA CALIDAD DEL SERVICIO EDUCATIVO DE LAS INSTITUCIONES UNIVERSITARIAS
}

\section{THE EXTERNAL INTERNATIONAL PEERS ASSESSORS OF THE QUALITY OF THE EDUCATIONAL SERVICE OF THE UNIVERSITY INSTITUTIONS}

\author{
Ana María Gutiérrez HubY* \\ Docente Principal de la Facultad de Ciencias Contables de la UNMSM \\ Elsa Violeta Rafael Díaz ${ }^{* *}$
}

[ReCEPCión: Abril De 2009 / Conformidad: MAYo de 2009]

\section{RESUMEN}

El objetivo del presente artículo es ofrecer mayores alcances respecto a los pares evaluadores externos y destacar la relevancia de su actuación dentro de un proceso de acreditación institucional. En este marco fueron considerados apartados como su misión, sus principios, capacidades y competencias en su ámbito de acción, su rol como elemento del órgano acreditador, entre otros aspectos interesantes que conviene tener en cuenta para enfrentar con mayores posibilidades una evaluación externa. Abordar el tema implicó la revisión de material documental publicado tanto por importantes entidades acreditadoras latinoamericanas que se valen del concurso de este equipo de profesionales para validar técnicamente el servicio educativo que prestan los programas evaluados, así como también de instituciones universitarias que han realizado procesos de acreditación. La conclusión es la consideración de la idoneidad del perfil que deben mantener estos especialistas para ser considerados como tales.

Palabras clave: Par externo, evaluación, acreditación.

\begin{abstract}
The goal of this article is to offer a further scope with regard to the external peer evaluators and to emphasize the relevance of their action within a process of institutional accreditation. In this framework it was considered as paragraphs, their mission, their criterion, their abilities and skills in the area of action, their role as a part in the accreditation organization, among other interesting aspects to have in consideration facing- with a range of possibilities- an external evaluation. Approaching the topic implied the research of documentary material published by well-known Latin-American accreditation entities that draw upon the collaboration of work teams in order to technically validate the educational service that is provided by the evaluated programs, as well as university institutions that experienced previous processes of accreditation. The resulting conclusion is then, the consideration of the suitable profile that these specialists must maintain to be considered as such.
\end{abstract}

Key words: External peer, evaluation, accreditation.

\footnotetext{
* Magíster en Contabilidad y Licenciada en Ciencias Administrativas de la UNMSM. Jefe de la Oficina de Calidad Académica y acreditación. E- mail: anamaria_0105@hotmail.com

**Colaboradora. E- mail: evrd107@yahoo.es
} 


\section{INTRODUCCIÓN}

Para las instituciones de educación superior universitaria a nivel de Latinoamérica, la acreditación constituye una verdadera exigencia tanto por el desafío que representa el promover y asegurar permanentemente la calidad del servicio educativo, así como por la demanda de transparencia, confiabilidad y garantía que es expresada por parte de los diversos usuarios del sistema.

En el Perú, todavía son pocas las instituciones universitarias que cuentan con programas académicos acreditados internacionalmente, aquí son las universidades privadas las que ejercen el liderazgo, entre ellas, podemos mencionar a la Universidad Católica y la Cayetano Heredia; en el caso de las universidades públicas ninguna aún ha acreditado internacionalmente algún programa académico, aunque es pertinente precisar que su universidad más representativa, San Marcos, tiene ya encaminado su primer proceso de acreditación internacional en su programa académico de Contabilidad y la gran mayoría de sus programas académicos estén inmersos en procesos de autoevaluación interna, con vistas a su mejora y posterior acreditación. $\mathrm{Al}$ respecto, sería importante acotar que aún al interior de estas instituciones universitarias es necesario que se siga incidiendo en la tarea de una sensibilización, enfocada en la unificación de una visión integradora de mejora de la calidad del servicio educativo.

El propósito del presente artículo no es detenernos en definir su conceptualización, sus características, reseñar su origen, ni explicar las razones de la lentitud del avance de estos procesos a nivel país, pues de ello ya nos ocupamos en un capítulo de precedente publicación, más bien lo que sí existe es el interés de abordarla bajo un enfoque orientado a su trascendencia como proceso de aseguramiento de la calidad, es decir, como mecanismo de avance hacia la excelencia y rendición de cuentas a la sociedad.

Bajo esta consideración, en el marco del aseguramiento de la calidad en el que se desenvuelve el referido proceso de acreditación, ésta no puede darse desconectada de un elemento imprescindible que es la evaluación, pues es a través de este ejercicio sistemático que ella se basa para mejorar la calidad de los programas evaluados. Así pues debe considerarse que la acreditación consistiría en un procedimiento cuyo objetivo es comparar el grado de acercamiento del objeto analizado con un conjunto de normas previamente definidas e implantadas como deseables, ello muy aparte de su implicancia en el reconocimiento público de que un programa satisface determinados criterios de calidad y por lo tanto es confiable.

La evaluación entonces debe ser desarrollada de forma sistemática para constituirse en el instrumento de revisión y generación de calidad, es decir, que en ella deben concurrir actividades organizadas de recolección, procesamiento, análisis de información y ser contrastados con propósitos y criterios de evaluación previamente definidos, de no ser así, las decisiones no contarán con una base sólida que las legitime y fundamente, ni permitirá la formación de una base de antecedentes disponibles para la gestión.

La evaluación aporta elementos de juicio sobre características y cualidades de los programas evaluados para así determinar el grado de calidad con que se cumplen funciones $\mathrm{y}$ tareas educativas.

Los aspectos evaluados son confrontados con un patrón de comparación que no es más que un estado deseable con el cual se contrasta el desempeño del programa o unidad objeto de evaluación, los que usual- 
mente son considerados son los patrones de evaluación interna y externa. En este orden se requiere que el programa evaluado realice una evaluación interna y luego una externa, esta última, destinada a validar y complementar la primera.

La evaluación externa usualmente está a cargo de una agencia acreditadora, quien encomienda a una comisión de pares evaluadores el ejecutar la visita y la evaluación, en el terreno de la unidad o programa evaluado en cuestión, configurándose esta fase de singular preponderancia, pues aporta el carácter de neutralidad, profesionalización y especialización que supone la participación de los pares evaluadores.

Por la relevancia que tiene esta fase en el proceso de acreditación, se le atribuye la connotación de constituir un proceso esencialmente externo a la institución que contará con mayor validez y legitimidad social cuánto más externo sea percibido.

\section{LA EVALUACIÓN EXTERNA EN EL CONTEXTO DE UN SISTEMA DE ASEGURAMIENTO DE LA CALIDAD Y ACREDITACIÓN}

Muchas son las definiciones halladas respecto a la evaluación externa, sin embargo, la que se presta con mayor pertinencia para abordarla en un contexto de aseguramiento de la calidad como es el objetivo del presente apartado, es la que nos brinda la Agencia Nacional de Evaluación de la Calidad y Acreditación (ANECA), quien nos refiere que "la evaluación externa consiste en la realización de un diagnóstico metódico e independiente para determinar si las actividades y los resultados relativos a la calidad de un programa o institución evaluado cumplen las disposiciones previamente establecidas y si éstas están implantadas de forma efectiva y son adecuadas para alcanzar los objetivos fijados".

Esta definición concuerda plenamente con el sentido de la acreditación cuyo objetivo principal es precisamente buscar el aseguramiento de la calidad de los programas evaluados, tal como fuera expresado en el apartado de la introducción del presente artículo, no obstante es preciso detenernos en los enunciados de la definición dada por esta importante agencia acreditadora, en procura de comprenderla cabalmente.

En este sentido, diremos que ciertamente es un "diagnóstico metódico e independiente" porque en su ejecución se ponen en práctica procedimientos formales para la evaluación de los elementos que integran el modelo aplicado por el comité evaluador externo, dándose aquí su connotación de "independiente", por cuanto la intervención de este equipo de profesionales aporta la transparencia e independencia requerida.

Por otra parte, cuando refiere que permite "determinar si las actividades y los resultados relativos a la calidad de un programa o institución evaluada cumplen las disposiciones previamente establecidas" y si estas mismas "están implantadas de forma efectiva y son adecuadas para alcanzar los objetivos fijados", cabe la reflexión de que en efecto el comité evaluador externo designado por el ente acreditador, se convierte en un medio para establecer mecanismos preventivos que faciliten la mejora continua del programa evaluado, mecanismos que nos remiten al sistema de regulación del sistema de educación superior, que no es otra cosa que el conjunto de todos los procedimientos, actividades, mecanismos e instrumentos destinados a asegurar la calidad del programa evaluado, objetivo primordial de la acreditación, de aquí la vinculación entre sí. 
Es preciso acotar la recomendación de los expertos y con la cual se coincide plenamente en cuanto a que su aplicación debe darse en las dos modalidades convencionales puesto que ambas se complementan entre sí, y fortalecen a la institución o unidad evaluada pues apoyan su desarrollo. Las dos modalidades de regulación aplicadas son: la regulación interna o autorregulación, es decir la ejecutada por la propia institución o programa académico y otra extra institucional dirigida por la agencia acreditadora quien finalmente otorga la certificación pública de la calidad corroborada.

\section{ACTUACIÓN DEL COMITÉ DE PARES EVALUADORES EXTERNOS EN EL MARCO DEL PROCESO DE ACREDITACIÓN}

La etapa de evaluación externa demanda el concurso de un grupo de evaluadores adecuadamente calificados cuya idoneidad e independencia se encuentre debidamente garantizada a través de los procedimientos de identificación y designación de pares que utiliza el organismo acreditador. Estos profesionales conforman el Comité de Pares evaluadores externos y su actuación constituye uno de los ejes primordiales en los que se apoya el proceso de acreditación.

Su labor consiste básicamente en comprobar de forma evidente, cómo se encuentra la unidad o programa evaluado, respecto al modelo de evaluación específico que se aplique y determinar su grado de ajuste al perfil de calidad establecido en los estándares que fija el modelo.

Para ello revisa, verifica y analiza la información contenida en el informe de autoevaluación previamente elaborado y alcanzado por la unidad o programa. El resultado de su actuación o conclusiones es plasmado en el informe de evaluación externa y/o la emisión del dictamen de recomendación de acreditación o no acreditación, el cual es alcanzado a la entidad acreditadora para la emisión de la Resolución o dictamen final de acreditación.

\section{FUNCIONES ENCOMENDADAS AL COMITÉ DE PARES}

La revisión documental respecto a las funciones del Comité de Pares Externos nos remite a las siguientes funciones básicas:

- Evaluar el grado en el que la carrera o programa se ajusta a los criterios y parámetros establecidos;

- Evaluar el grado de cumplimiento de los propósitos que la carrera o programa se ha fijado; $y$,

- Validar el informe de autoevaluación. La autoevaluación ejecutada por la unidad o programa académico se encuentra incompleta hasta que es validada por los pares evaluadores externos. El comité de pares le aporta la mirada externa y un juicio calificado.

\section{CONSIDERACIONES ESPECÍFICAS EN EL DESEMPEÑO DE LOS PARES EVALUADORES}

Los pares evaluadores externos deberán acatar las siguientes obligaciones:

- Integrar el comité de pares y trabajar en equipo con sus colegas de diversa pertenencia institucional y disciplinar.

- Respetar las pautas establecidas en el código de ética del organismo acreditador que los obliga a actuar con independencia de criterio, sin asumir la gestión 
de intereses ajenos a la tarea evaluadora. Los pares están obligados a excusarse de intervenir en los casos en que, por razones de vínculos académicos, institucionales o familiares, o bien de amistad o enemistad personales, pudiera estar comprometida su imparcialidad. Mediante la firma de un convenio de confidencialidad, los pares evaluadores se comprometen a cumplir con las normas de dicho Código y a no divulgar las informaciones contenidas en las solicitudes de acreditación evaluadas por ellos ni el resultado de la evaluación.

- Evitar la expresión de opiniones personales y/o grupales o bien de juicios evaluativos anticipados durante la visita a las instituciones a evaluar.

- Basar todas las decisiones en los criterios y estándares establecidos en el modelo de evaluación aplicado y los acuerdos generales de su aplicación, con la mayor objetividad posible.

- Realizar el trabajo de evaluación con el apoyo técnico del equipo profesional de la entidad acreditadora, quien realizará la coordinación técnica de las evaluaciones y estará a disposición de los pares ante las dudas o preguntas que puedan surgir a lo largo de todo el proceso evaluativo.

- Redactar el dictamen para la carrera evaluada.

\section{FASES PUNTUALES DEL TRABAJO DE LOS PARES EVALUADORES}

Resumiendo las dinámicas de trabajo establecidas por las entidades acreditadoras, mencionamos las siguientes etapas que pautean el trabajo de los pares evaluadores:

\section{Primera etapa: \\ Estudio preliminar, reunión del Comité y preparación de las visitas de campo}

Constituye la etapa preparatoria en la que se determinan los principales aspectos de atención y análisis, actores para entrevistar, actividades que se realizarán, el diseño de la agenda que puede incluir entrevistas a autoridades, profesores, alumnos observación de clases visita a talleres, en suma, es una planificación de la visita.

\section{Segunda etapa: \\ Ejecución de la visita a la unidad que ha solicitado su evaluación}

En esta segunda etapa los pares realizan la visita de campo a la unidad académica con el propósito de revisar el informe de autoevaluación presentada por la unidad o programa, registrar observaciones y realizar constataciones (revisión documental) y entrevistas con autoridades, docentes, alumnos u otros actores que aporten a la elaboración de juicios evaluativos.

La visita demanda un tiempo estimado entre dos a tres días.

\section{Tercera etapa:}

Redacción de la propuesta preliminar de dictamen.

Una vez evaluados todos los aspectos de la autoevaluación ejecutada por la unidad o programa, los pares trabajan en la redacción de la propuesta preliminar de dictamen, incorporando en ellos todas las observaciones y conclusiones que se desprendan de la visita.

Cabe indicar que la mayoría de entidades acreditadoras tienen como política no adelantar ninguna opinión u observación a la unidad evaluada. 


\section{FORMACIÓN DE LOS PARES EVALUADORES Y SU DESIGNACIÓN COMO TALES}

Con el objeto de conseguir grupos de evaluadores con la profesionalidad que requieren los procesos de valuación externa, los organismos acreditadores efectúan una convocatoria de profesionales para que participen en esta etapa de los procesos de acreditación.

Esta convocatoria va dirigida a profesores de universidad, personas relacionadas con los procesos de evaluación y auditoría, con la calidad, con los campos científicos-técnicos y carreras por evaluar.

Los profesionales interesados tienen la oportunidad de remitir sus solicitudes con las formas establecidas por el órgano acreditador convocantes, expresando su disponibilidad para participar en los procesos de evaluación externa.

Con el propósito de conseguir formar y desarrollar las competencias necesarias que les permitan a estos profesionales el ejercicio de las funciones de evaluador externo en términos de profesionalidad, eficacia y transparencia, el organismo acreditador desarrolla su programa de formación de evaluadores. La capacitación es impartida por los pares evaluadores con mayor experiencia del órgano acreditador.

El objetivo es constituir grupos de evaluadores con capacidad para realizar el contraste externo necesario en los procesos de evaluación institucional, certificación y acreditación, en condiciones de independencia y profesionalidad.

La estructura o dinámica de la formación que se imparte es diversa, dependiendo ésta de la política de la entidad acreditadora convocante; algunas entidades por ejemplo, consideran impartirlo por módulos virtuales y presenciales; virtuales, en el que los aspiran- tes a evaluadores obtendrán los conocimientos teórico-prácticos de calidad universitaria $y$, presenciales, en el que la formación se centra en una simulación de evaluación externa para adquirir las habilidades competencias propias de ambos enfoques evaluativos; no obstante, otras entidades únicamente consideran la modalidad presencial con el objetivo de hacer conocer y manejar la metodología aplicada en la evaluación de programas académicos, considerando también el ejercicio práctico de analizar e interpretar el modelo de evaluación.

\section{ALGUNOS REQUISITOS Y VIGENCIA PARA EL EJERCICIO COMO EVALUADOR DEL ORGANISMO DE ACREDITACIÓN}

Algunas entidades acreditadoras consideran que la condición de evaluador tiene una validez de cinco años renovables, a lo largo de los cuales, la agencia acreditadora actualizará los conocimientos de sus evaluadores y auditores. Transcurrido los cinco años, el evaluador interesado en prorrogar la vigencia de su condición, deberá someterse nuevamente a la evaluación de su currículum y desempeño.

Otras entidades acreditadoras, como la Comisión Nacional de Acreditación de Pregrado de Chile, mantienen un registro de expertos para cada carrera o área de carreras y por categorías. Los especialistas considerados en el registro deben cumplir como norma general con tener por lo menos diez años de ejercicio profesional, ser reconocidos como expertos en su área y recomendados por un representante de una institución de reconocido prestigio en el ámbito de la profesión o disciplina. Asimismo, deben estar dispuestos a cumplir las exigencias propias del órgano 
acreditador, incluyendo la participación en los talleres de formación que esta realiza. Dicho registro está colocado en la página web institucional para su consulta por las instituciones y público en general, interesado.

\section{COMPORTAMIENTO ÉTICO DE LOS EVALUADORES EXTERNOS}

Todas las entidades acreditadoras tienen establecido un modelo de código de ética particular para los evaluadores externos, ello bajo la consideración de que la evaluación externa debe realizarse en un marco de probidad, honradez, transparencia, respeto a la institución evaluada y alto compromiso ético; con este objetivo, se busca regir el comportamiento y las relaciones de los evaluadores con sus pares al interior del Comité de Evaluación Externa, con los miembros de la colectividad institucional y con el medio externo durante el proceso de evaluación y luego de él. A continuación sólo mencionaremos aquello aspectos en los que todos ellos coinciden.

\section{En lo que respecta a su imparcialidad e independencia}

- El par académico o evaluador externo, por su condición de docente universitario seleccionado por la entidad acreditadora en base a sus méritos y su ética de profesional, es responsable de mantener un criterio libre de conflicto de intereses e imparcial. Es decir se le demanda conducirse con independencia de criterio en el proceso de evaluación, sin asumir la representación de intereses ajenos legítimos o no.

- No deberá aceptar responsabilidades en las que se encuentre limitada su indepen- dencia mental. Este punto es entendido en el sentido de que los profesores universitarios deberán abstenerse o excusarse de participar como evaluadores externos en los siguientes casos:

- Cuando sea cónyuge o pariente de alguna autoridad o miembro de la institución que se evalúa.

- Cuando tenga o haya tenido alguna relación económica con la institución que se evalúa.

- Cuando tenga relaciones o intereses que le pudieran impedir su independencia.

- Haber mantenido relaciones laborales o contratos con la institución evaluada en los cinco años anteriores a la evaluación externa.

En estos casos se establece que no hay independencia mental. Lo que generalmente se estila por parte de las agencias acreditadoras es que el par evaluador externo firme una declaración jurada de no estar incurso en estas prohibiciones.

\section{Con respecto a la calidad que debe evidenciar en su función de evaluador}

- Se espera del evaluador un trabajo profesional actuando cuidadosamente con el entrenamiento, capacidad y experiencia necesarios para su labor.

- Deberá tomar el curso de formación de evaluadores impartido por la entidad acreditadora.

- Su trabajo, informes y opiniones deberán fundarse en hechos reales sin ocultar o desvirtuar hechos que induzcan a error para la toma de decisiones.

- El evaluador externo actuará sin prejuicios acerca de las formas diversas en que se desarrolla la educación superior, los 
cuales pueden sesgar su análisis y los resultados de la evaluación externa sobre el desempeño de una determinada institución, programa o carrera en cuya evaluación participa.

- Deberá comprender y respetar los aspectos relativos a la cultura, los principios y estilos de organización y trabajo de la institución, carrera o programa en cuya evaluación participa, distanciándose para ello de las características de su institución de procedencia así como de sus criterios personales sobre estos aspectos. Este aspecto es particularmente importante dadas las diferencias culturales, regionales, de propósitos y de estilos de organización entre las instituciones de educación superior.

- Deberá cimentar la calidad de su trabajo en la honradez, laboriosidad y capacidad profesional.

- El evaluador externo que se encuentre en alguna situación o circunstancia que no le permita desarrollar su trabajo correctamente deberá comunicar oportunamente a la entidad acreditadora.

\section{Con respecto a la confidencialidad}

- Al evaluador externo no le está permitido revelar, en beneficio propio o de terceros los resultados de la evaluación realizada a ninguna persona de la institución evaluada. La comunicación de los resultados oficiales de la acreditación es facultad exclusiva de la entidad acreditadora. Es decir, que el evaluador tiene prohibido emitir juicios en forma individual, así como recomendaciones acerca de la institución, programa o carrera durante la evaluación externa y después de ella.

- Se podrán intercambiar impresiones, criterios u opiniones pero no deberán darse datos que identifiquen a otras instituciones evaluadas como referencia.

- Se prohíben comunicaciones o difusiones que hagan los evaluadores en alusión a sus honorarios ni se podrán comparar con los de otros colegas.

\section{Con respecto a la honradez}

- Faltará a la dignidad profesional todo evaluador que de manera directa o indirecta intervenga en arreglos o asuntos que no cumplan con la moral y honradez profesional.

- Ningún evaluador podrá obtener ventajas económicas por su trabajo que no sea otorgado por la entidad acreditadora.

- En ningún caso se podrán recibir comisiones o gratificaciones por parte de la institución evaluada. Aquí cabe acotar que no les está permitido a los pares integrantes de la Comisión de Evaluación Externa:

- Aceptar obsequios

- Aceptar invitaciones, almuerzos, recepciones y otras actividades.

- Aceptar traslados a centros de diversión.

- Atender citaciones de las autoridades de la institución evaluada.

- El evaluador no deberá solicitar con fines personales, documentos de la institución.

\section{Con respecto a la lealtad}

- El evaluador no deberá aprovecharse de situaciones que pudieran perjudicar al órgano acreditador en beneficio propio.

- No deberá ofrecer ni aceptar trabajo directa ni indirectamente de parte de las instituciones asignadas si no es con previa autorización del ente acreditador. 


\section{Con relación al respeto}

- El evaluador cuidará sus relaciones personales con sus colegas y con las instituciones evaluadas a los que sea asignado, buscando que nunca se menoscabe la dignidad del órgano acreditador sino que la enaltezca actuando con espíritu de servicio y de trabajo en equipo.

- No deberá hacer comentarios sobre otro evaluador de la institución asignado que perjudiquen su reputación o su prestigio.

- El evaluador debe participar en la enseñanza a otros colegas de los conocimientos adquiridos, manteniendo las más altas normas profesionales y de conducta en su enseñanza.

- Debe mantenerse actualizado en los conocimientos relativos a su actividad.

\section{Con respecto a las sanciones y la pérdida de su condición de evaluador}

La interrupción total o parcial del ejercicio de la calificación de evaluador o auditor puede deberse a diferentes causas y con efectos diversos.

- Con carácter general, el evaluador externo perderá dicha condición con carácter definitivo cuando actuase fuera de las normas yguías establecidas por el ente acreditador, bien por causas alevosas o bien derivadas de desconocimiento, pasividad o pérdida de la capacidad profesional, o bien cuando la entidad acreditadora tuviese conocimiento de actuaciones, omisiones o cualquier otra circunstancia que convirtiese en falsos con carácter objetivo los rasgos aportados por el evaluador cuando postuló a la condición obtenida.

- El incumplimiento del código de ética por parte de cualquiera de los miembros del Comité de Evaluación Externa lo hará acreedor a la sanción que le imponga el órgano acreditador, de acuerdo a su gravedad y a la afectación del prestigio del mismo.

- Según la gravedad de la falta, la sanción podrá consistir en:

- Amonestación verbal

- Amonestación por escrito

- Suspensión temporal como evaluador

- Expulsión

\section{CONCLUSIONES}

En el contexto de la calidad, la evaluación constituye el mecanismo utilizado por las instituciones para legitimar las decisiones adoptadas con fines de mejoramiento.

Podemos concluir que el instrumento de la acreditación es la evaluación previa, la misma que tiene por objetivo determinar el grado de aproximación de los aspectos evaluados con los estándares establecidos por el organismo acreditador.

Cuanto más externo sea percibido el proceso de acreditación por parte de la comunidad académica, mayor será la percepción de objetividad y legitimidad del proceso, por lo que resulta relevante la heterogeneidad de procedencia del comité evaluador pues ello le dará una connotación de transparencia e independencia al proceso.

Aunque la capacitación del cuerpo de evaluadores externos es un aspecto altamente considerado por cada organismo acreditador, la conducta ética que éstos demuestren lo es aún más, dependiendo de ello su prestigio e integridad, de ahí la preocupación por lograr la máxima concientización de estos profesionales para que sus funciones de evaluación sean ejecutadas con inclinación a la honestidad, legalidad y justicia. 


\section{REFERENCIAS BIBLIOGRÁFICAS}

1. Consejo Nacional de Acreditación CNA. (2003). Protocolo para el informe de evaluación externa con fines de acreditación institucional. Colombia.

2. Comisión Nacional de Acreditación de Pregrado - CNAP. (2001). Manual para el desarrollo de procesos de autoevaluación. Chile.
3. Consejo de Acreditación en la enseñanza de la contaduría y administración - CACECA. (2009). Manual de Curso Taller "Formación de Pares Evaluadores". México.

4. Universidad Nacional Mayor de San Marcos. Oficina de Calidad Académica y Acreditación. (2003). Lineamientos para una politica de calidad, autoevaluación y acreditación. Perú. 